\title{
A study on the Horizontal Control of a Seat for Heavy Machinery using a Phase Adjusting Technique
}

\author{
Chae-sil. Kim ${ }^{1, a}$, Min-jae. Shin ${ }^{1}$, Jae-min. Kim ${ }^{1}$ and Chang-min. Keum ${ }^{1}$ \\ ${ }^{1}$ Changwon National University, Department of Mechanical Engineering, 641-773 Changwondaehark-ro Uichang-gu Changwon-si \\ Gyeongsangma-do, KOREA
}

\begin{abstract}
This study proposes a dynamic plant model to simulate the performance of a heavy equipment vehicle seat system. The system controls the transmission of vibration using a phase adjustment control method. First, a dynamic model for the flexible heavy equipment seat is proposed using a commercial multi-body dynamic analysis tool, ADAMS. This model is used as a plant model. Then a phase adjustment control mechanism for reducing the vibration and shock transmitted by the seat is applied to the driving dynamics control plant model. Angle control using the phase adjustment control was confirmed. This technique is expected to be the basis for a future commercial system.
\end{abstract}

\section{Introduction}

Heavy equipment such as earth-moving machines are widely used on construction sites, but the frame of the heavy equipment typically does not have a device to absorb vibration. As a result, more than $90 \%$ of the vibrations and impacts that occur due to the operation of the engine and the equipment is transmitted to the equipment operator.

Typically, vibrations of $1 \mathrm{~Hz}$ or less can cause motion sickness. Even when working for a short time with eyes trembling in the $30 \mathrm{~Hz} \sim 80 \mathrm{~Hz}$ range, the driver is likely to feel fatigue. With long exposure, health and work efficiency can be significantly impacted.

Exposure to heavy equipment vibration has also emerged as a factor in serious industrial accidents.

To reduce industrial accidents and to provide the best working environment for heavy equipment operators, the development of heavy equipment seats with various additional controls, as shown in Fig. 1, has attracted increased attention in studies in related fields.

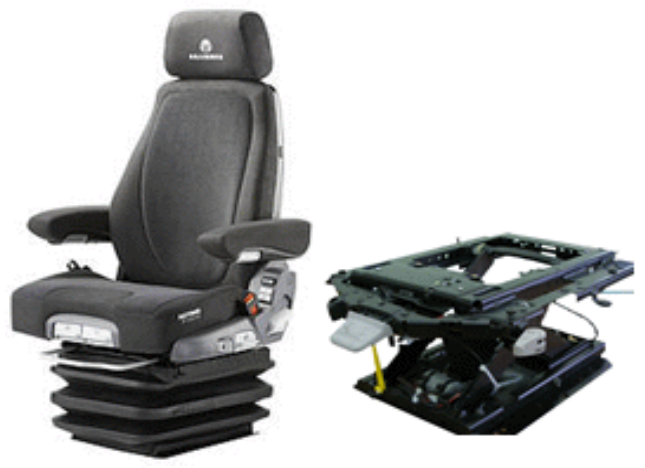

Figure 1. Heavy equipment seat

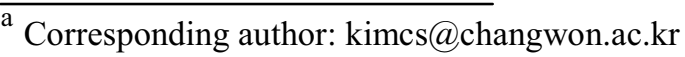

In order to reduce the transmission of vibration and impacts to the operator seat of heavy equipment, which can affect the operator's comfort and functionality, the seat requires a variety of features. Various manufacturing and control techniques have been studied to reduce the vibration and impact. Because of the narrow space in the cockpit, as well as the need for access for equipment repair, the seat should also have sliding and reclining features.

However, conducting empirical testing to collect performance data on heavy equipment seats typically involves high cost, and therefore the development of a simulation technique would be beneficial.

This study first proposes a dynamic model for simulating a flexible heavy equipment seat using a commercial multi-body dynamic analysis tool, ADAMS. This model is used as a plant model.

Then, the driving dynamics control plant model is used to introduce a phase adjustment control mechanism, in order to reduce the vibration and shock transmitted through the seat.

Finally, the horizontal control of the heavy equipment sheet is checked.

\section{Multi-body dynamics plant modelling of a heavy equipment seat [5]}

For modelling of the heavy equipment seat, this study used a commercial tool, ADAMS multibody dynamics analysis. Fig. 2 illustrates the proposed process used to establish a multi-body dynamics model of the heavy equipment seat control. 


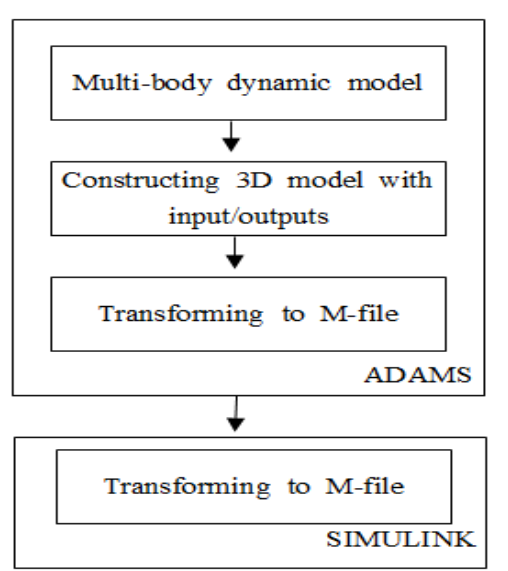

Figure 2. Procedure for constructing the dynamic plant model.

First, in order to analyze the vibration characteristics of the suspension seat, a multi-body dynamics analysis model was constructed.

Next, the dynamics analysis model was applied to define and apply the details of the loading and boundary conditions, and the movement mechanism, to reproduce the behavior of real models.

Finally, after creating an M-file in MATLAB, the input and output for the heavy-duty seat were defined in the ADAMS control module for the multi-body dynamics model.

This was applied to the plant model for vibration control in SIMULINK.

\subsection{Multi-body dynamics plant modelling of the heavy equipment seat [4]}

The seat suspension is the component that has the greatest impact on the vibration of the insulating seat. For this purpose, springs and dampers were employed to isolate the vibration. The initial tension of the spring can be set with a weight adjustment knob.

The seat was also designed with a link mechanism for realizing movement in the vertical direction, to allow the operator to adjust the height of the seat.

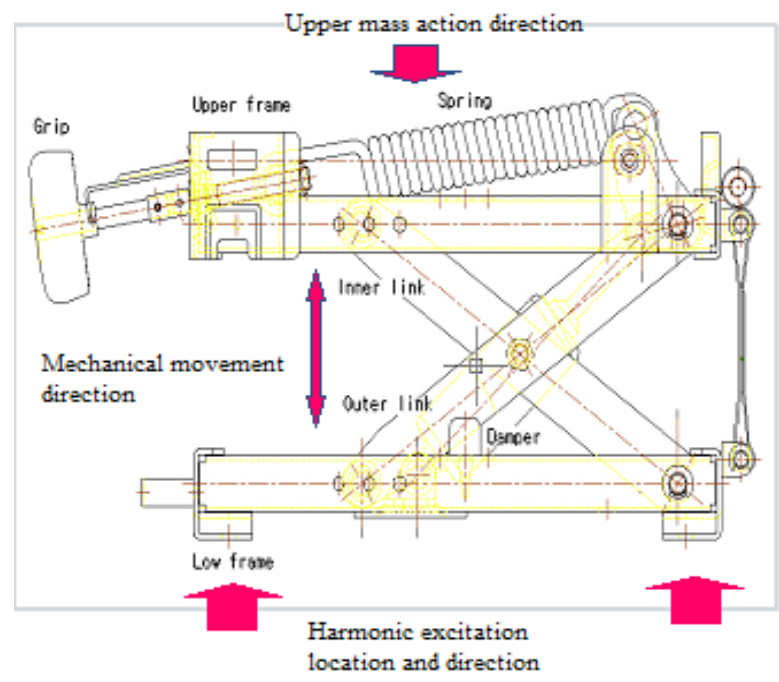

Fig. 3 is a schematic diagram of the seat suspension and shows the operating mechanism. Fig. 4 shows a multi-body dynamic model of the proposed heavy equipment seat produced with the ADAMS multi-body commercial analysis program.

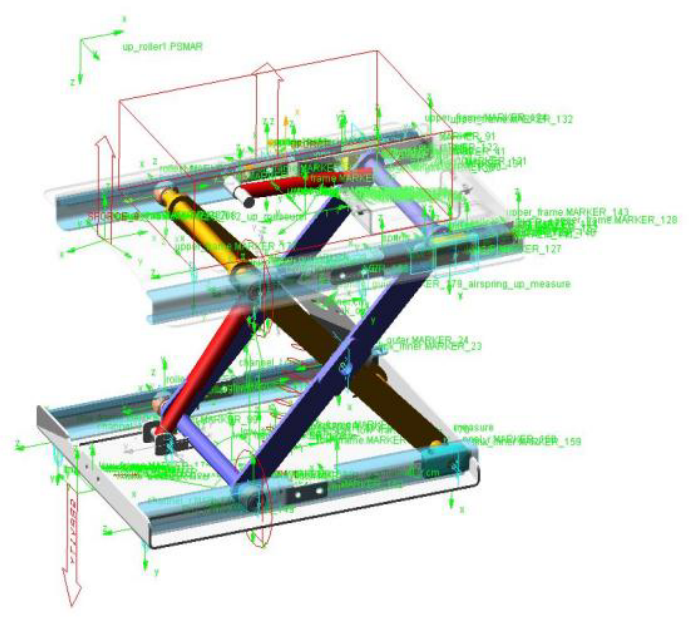

Figure 4. Dynamic analysis model of the suspension seat created using ADAMS

The relative motions of translational joints, revolving joints and fixed joints were established using a point redemption, translation, rotation, to set the conditions needed to reproduce a real working mechanism.

The load condition was set in the -Z-axis direction. The $-\mathrm{z}$ direction is the vertical direction under gravity conditions.

\subsection{Heavy equipment seat plant model}

Input and output variables of seat model are defined in "Plant input..." and "plant output..." of "control toolkit" module. In this study, for horizontal control, two input parameters in the $\mathrm{X}$ axis direction and the $\mathrm{Y}$-axis direction were measured from the center of the top frame and applied.

The 6 -output of the plant model were used to confirm translational displacement, angular velocity. A heavy equipment seat model was constructed to achieve horizontal control based on the vibration output.

6 measurement sensors were installed at intervals in the top frame center portion of the seat to determine the vibration characteristics. These were applied in the same manner as actually installed acceleration and gyro sensors.

Fig. 5 shows the "Adams sub" block of MATLAB in the "Control" "ADAMS / Controls plant export" of the module. This input and output of the multi-body dynamics model of the heavy-duty seat was configured to convert to the "Adams_sub" block.

Fig. 6 is the "Adams sub" block in SIMULINK. As shown in Fig. 5 it consists of two input parts and six output parts. This control, using the phase adjustment control method, was used as a model plant for the vibration control of the heavy equipment seat.

Figure 3. Suspension schematic diagram 


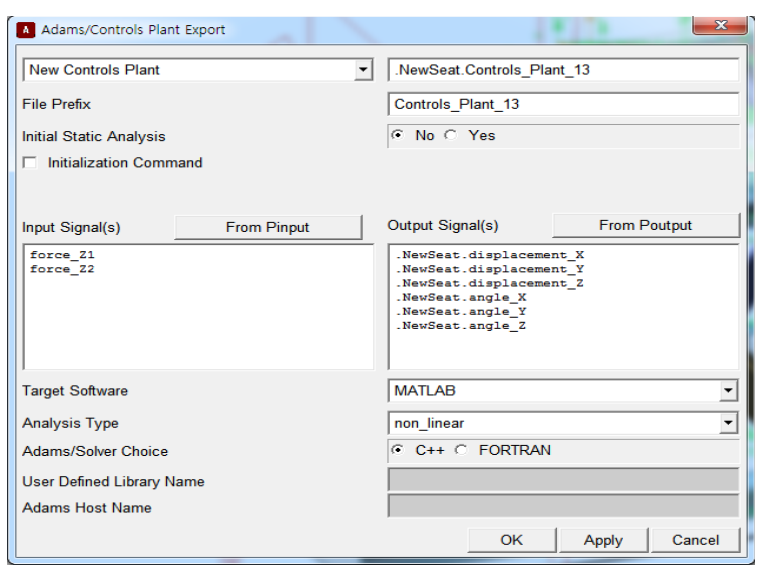

Figure 5. Procedure for converting to "Adams sub" block

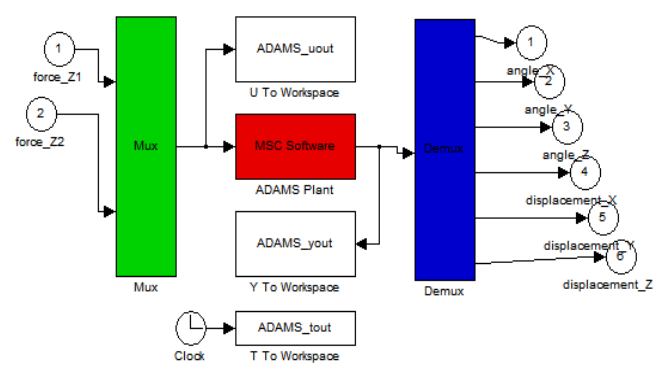

Figure 6. Detail "Adams sub" block in SIMULINK

\section{Phase adjustment control method}

The concept of control technique with adjusting phase presented by Jung, Jo, Kim and Cho [2]. As shown in a Fig. 7 and Fig. 8 is used.

The vibration may be diminished by adding control forces with a half period in the opposite direction to achieve the response shown in Fig. 8.

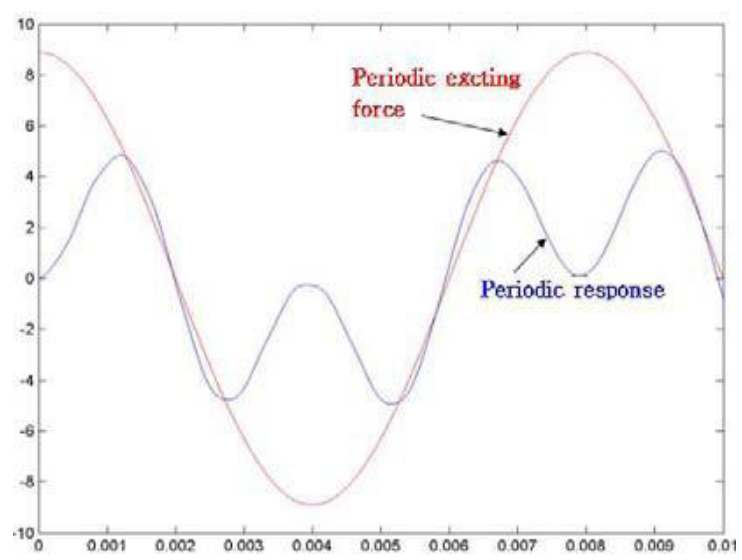

Figure 7. Periodical input and output from general

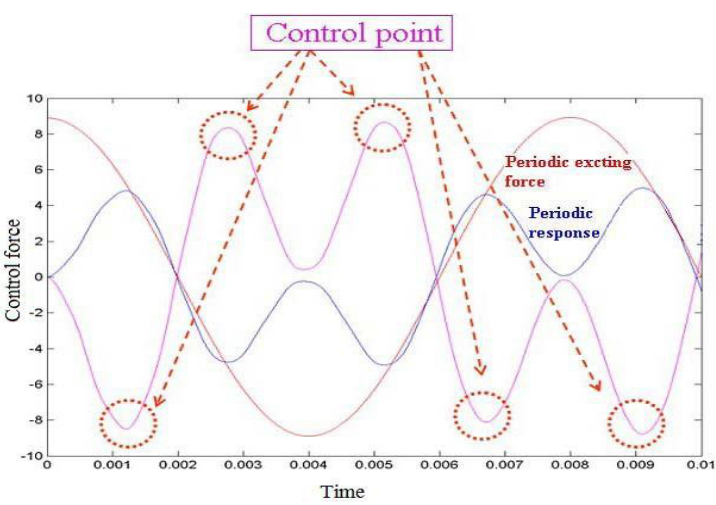

Figure 8. Forcing point for control and periodical response

In this study, vibration control of the heavy equipment seat focused on the use of this phase regulation method. A Phase controller constructed in SIMULINK is shown in Fig. 9.

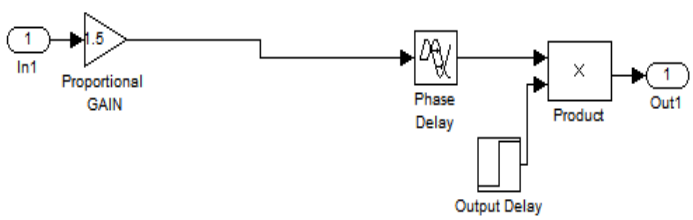

Figure 9. Phase adjuster block in SIMULINK

\section{Heavy-duty seat horizontal control simulation results}

As Fig. 10 presents the structured control model for the horizontal control of a heavy equipment seat using the phase adjustment control method.

To ascertain the control needed for the heavy equipment seat, the scope of SIMULINK was installed in six output section of control model and was composed feedback system applying output section value in order to control the horizontal.

Figs. $11 \sim$ Fig. 16 show the original output values before the input values were applied for control.

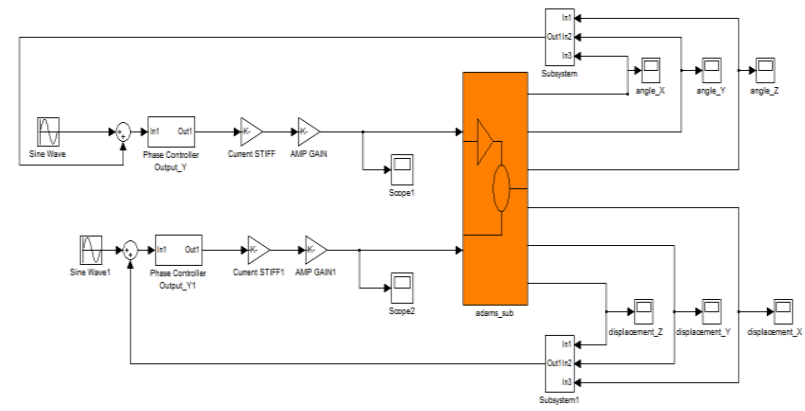

Figure 10. Active vibration control simulation model 


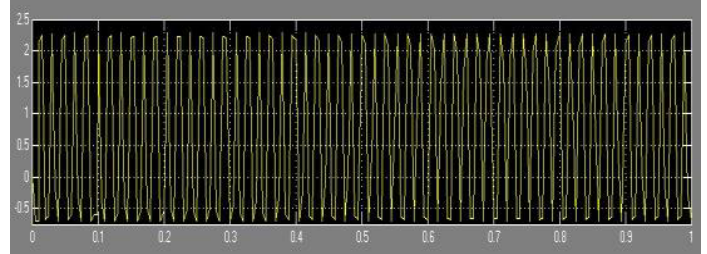

Figure 11. The angular displacement $\mathrm{X}$ before control

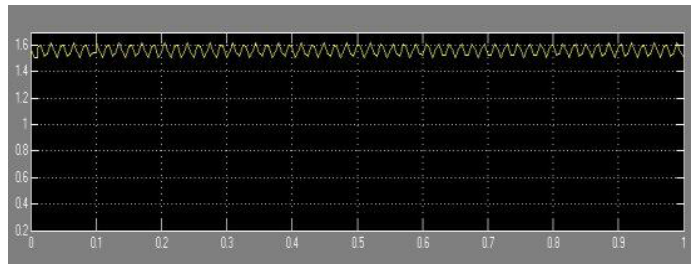

Figure 12. The angular displacement $Y$ before control

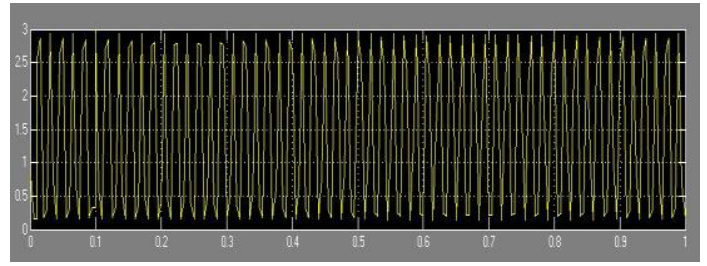

Figure 13. The angular displacement $\mathrm{Z}$ before control

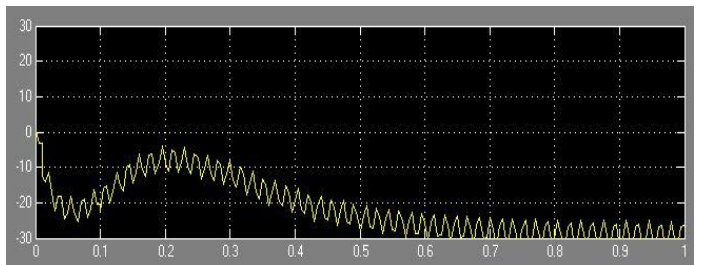

Figure 14. The displacement $X$ before control

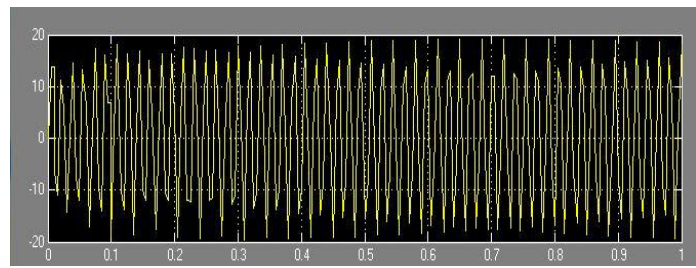

Figure 15. The displacement $Y$ before control

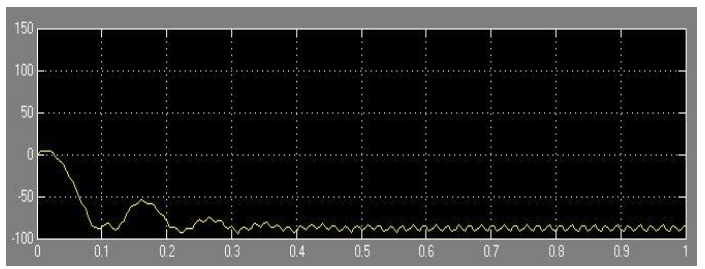

Figure 16. The displacement $Z$ before control

Figs. $17 \sim$ Fig. 22 show the output values when the input values have been applied. In this sequence, the input values were set to be applied every 0.2 seconds. Actual control may be achieved with a control frequency ranging from 0.4 seconds.

Figs. $17 \sim$ Fig. 19 confirm that the angle of the controlled heavy equipment seat is zero.
In conclusion, it was determined that it is possible to use the model of the heavy equipment seat with the phase adjustment control method to control the angle of the operator and dynamically maintain a horizontal work position.

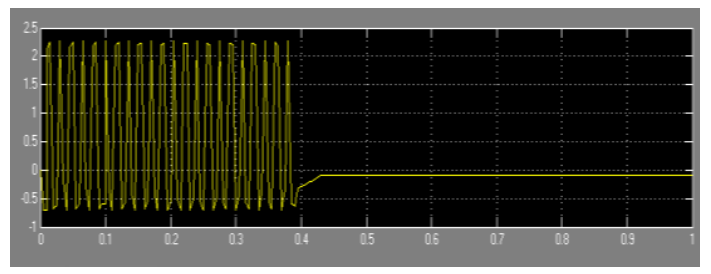

Figure 17. The angular displacement $X$ after control

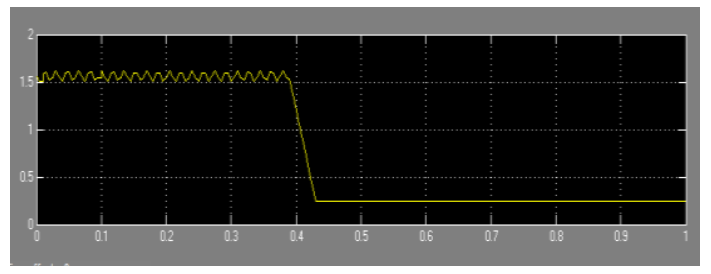

Figure 18. The angular displacement $Y$ after control

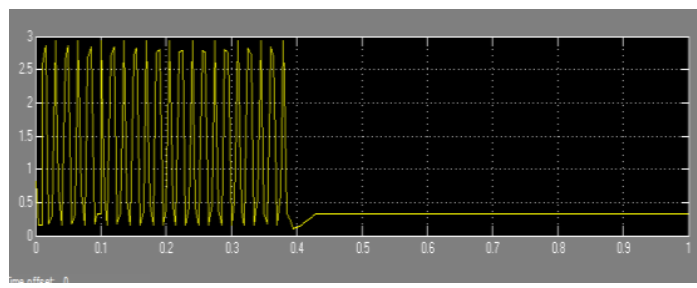

Figure 19. The angular displacement $\mathrm{Z}$ after control

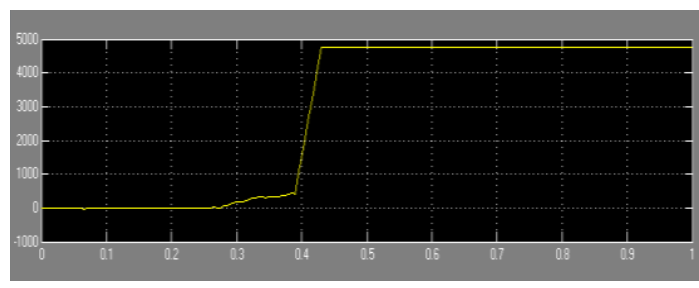

Figure 20. The displacement $X$ after control

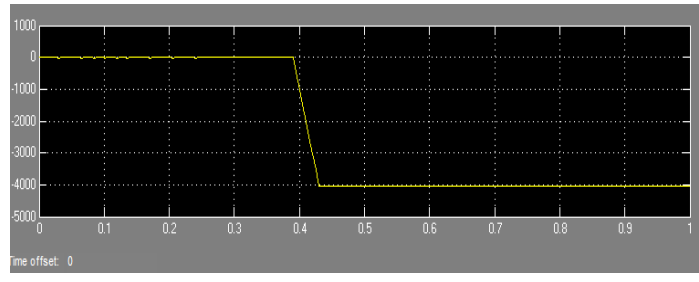

Figure 21. The displacement $Y$ after control

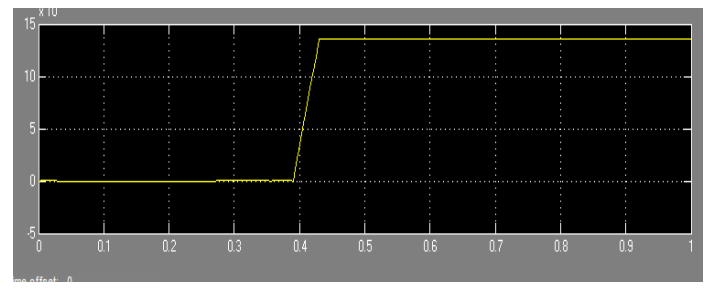

Figure 22. The displacement $\mathrm{Z}$ after control 


\section{Conclusions}

By the phase adjustment control, the angle of the controlled heavy equipment seat is zero but displacement was not controlled uniformly.

The results were as follows.

(1) A heavy equipment seat body dynamics model was constructed using a SIMULINK block.

(2) Angle control using the phase adjustment control was confirmed.

(3) it was determined that it is possible to use the model of the heavy equipment seat with the phase adjustment control method to control the angle of the operator and dynamically maintain a horizontal work position.

(4) Because displacement control was dissatisfaction, these results will be in a subsequent report.

(5) In order to control the vibration of the heavy equipment seat, the study suggested the potential use of a phase adjustment control method.

(6) This technique is expected to be the basis for a future commercial system.

\section{Acknowledgment}

Following are results of a study on the "Leaders in Industry university Cooperation" Project, supported by the Ministry of Education(MOE).

\section{References}

1. C. S. Kim, W. C. Lee, "A study on the Active Control for State Space Model of Flexible Rotors Using a Phase Control Method," Engineering Research \& Techonology, vol. 6, pp. 71-75, (2006).

2. H. H. Jung, H. M. Jo, C. S. Kim, S. Y. Cho, "MultiBody Dynamic Modeling for a Flexible Rotor and Vibration Control using a Novel Phase Adjusting Technique," Journal of KSMPE, vol. 10, pp. 87-92, Feb. (2011)

3. J. B. Na, W. C. Lee, H. H. Jeong, C. S. Kim, S. W. Kim and K. Y. Kim, "Control of the high speed flexible-rotor system using multi-body dynamic method,", in Proc. 2006 autumn KSPE Conf., pp. 265-266, (2006)

4. W. C. Lee, H. H. Jeong, S. Y. Kim, C. S. Kim, “A study Vibration characteristic of the Heavy Machinery Seat Multibody Dynamic Analysis Method," in Proc. 2007 spring KSPE Conf., pp. 117$118,(2007)$

5. C. S. Kim, M. J. Shin, J. M. Kim, C. M. Keum, “A Study on the Multi-Body Dynamic Control Plant Modeling of a Seat for Heavy Machinery," in Proc. 2015 autumn KSMPE Conf., pp. 88-89, (2015) 